\title{
CONVERGENCE TO EQUILIBRIUM OF A BODY MOVING IN A KINETIC SEA
}

\author{
XUWEN CHEN AND WALTER A. STRAUSS
}

\begin{abstract}
We consider a continuum of particles that are acted upon by an external force $\mathbf{G}(t, \mathbf{x})$ and that collide with a rigid body. The body itself is subject to a constant force $E$ as well as to the collective force of interaction with the particles. We assume that the particles that collide with the body reflect probabilistically with some probablility distribution $K(v, u)$. Under certain conditions on $\mathbf{G}(t, \mathbf{x})$ and $K(v, u)$, we identify an equilibrium velocity $V_{\infty}$ of the body and we prove that this equilibrium is asymptotically stable.
\end{abstract}

\section{INTRODUCTION}

In previous work of this type the particles have been subject to no force $(\mathbf{G}(t, \mathbf{x}) \equiv 0)$ and therefore travel with constant velocities between their collisions with the rigid body. In this paper we initiate an investigation of how some forces on the particles may affect the motion of the body. What is the asymptotic effect of such particle forces on the body? Our ultimate goal for the future is to treat a plasma in which the particles are charged and thus are subject to electromagnetic forces, which may be either external or created by the particles themselves. In this paper the forces are external.

Our problem has a free boundary, the moving location of the body. The other unknowns are the configuration and motion of the particles. The particles may collide with the body diffusely. Boundary interactions in kinetic theory are very poorly understood, even when the boundaries are fixed. Free boundaries are even more difficult. Our most important assumption is that the whole system, consisting of the body and the particles, starts out rather close to an equilibrium state.

We consider classical particles that are extremely numerous and subject to an outside force $\mathbf{G}$, which in this introductory study we assume to be small and decaying. While one could consider modeling the particles as a fluid, we instead model them as a continuum with a phase-space density $f$ as in kinetic theory 11, 14, 15. Furthermore, the interaction of the particles with the body at its boundary can be quite complicated in typical physical scenarios. For instance, the boundary may be so rough that a particle may reflect from it in an essentially random way. There could even be some kind of physical or chemical reaction between the particle and the molecules of the body. Therefore we model the collisions by a probability distribution $K$. This is sometimes called diffuse reflection. Altogether, the prescribed data consists of the initial density $f_{0}$, the force $\mathbf{G}$, the collision distribution $K$, and a constant force $E$ on the body.

The present paper is a sequel to [9, 10 and is also highly motivated by a series of remarkable papers 1, 5, 6. Most of the earlier papers, including 1, 5, 6, 9, 10, were devoted to proving more detailed behavior such as determining the precise rate of approach to equilibrium. In particular, the earliest papers [6, 5] assumed specular reflection at the free boundary. On the other hand, considering a perfect gas, the authors of 1 chose a Maxwellian-like distribution for the reflected particles. More generally, for the reasons mentioned above we considered in 9, 10 as well as in this paper a general probabilistic law of reflection. In 9, 10, where $\mathbf{G}=0$, we found a condition on $K$ and $f_{0}$ that is almost necessary and sufficient that the approach to equilibrium is reversed; that is, the approach begins from the right side, say, and ultimately approaches from the left. The present paper does not deal with such detailed behavior, focusing instead on whether it approaches equilibrium at all. Some numerical investigations, mostly dealing with a diffuse reflection which ejects Maxwellians, have appeared in 13, 16, 17, 18. Some other related investigations that deal with the speed of approach to equilibrium in the specular reflection case are $2,7,8,13$. See also the survey in 4 . In all of the preceding references, the particles are

Date: v2 for SIMA, 10/24/2015.

2010 Mathematics Subject Classification. 70F45, 35R35, 35Q83, 70F40.

Key words and phrases. kinetic, free boundary. 
identical, the body is initially moving near the equilibrium velocity $V_{\infty}$ and the motion of the body is one-dimensional, even though the particles move in three (or $d$ ) dimensions. The current paper is the first one for which the particles are themselves subject to a force $\mathbf{G} \neq \mathbf{0}$.

We now describe our problem in mathematical terms. For simplicity we take the body to be a vertical disk $D(t)$ centered at the point $(X(t), 0,0)$ and traveling with velocity $(V(t), 0,0)$. We assume there is a constant horizontal force $E \in \mathbb{R}$ acting on the body, in addition to the horizontal frictional force $F(t)$ due to all the colliding particles at time $t$. Thus

$$
\frac{d X}{d t}=V(t), \quad \frac{d V}{d t}=E-F(t)
$$

The particle distribution $f(t, \mathbf{x}, \mathbf{v})$ satisfies the Vlasov equation

$$
\partial_{t} f+\mathbf{v} \cdot \nabla_{\mathbf{x}} f+\mathbf{G}(t, \mathbf{x}) \cdot \nabla_{\mathbf{v}} f=0
$$

in $\mathbb{R}^{3} \backslash D(t)$. We assume the initial velocity $f(0, \mathbf{x}, \mathbf{v})=f_{0}(\mathbf{v})$ depends only on $\mathbf{v}$ and is even. At each collision with the body we assume the diffuse law of reflection

$$
f_{+}(t, \mathbf{x}, \mathbf{v})=\int_{\left(u_{1}-V(t)\right)\left(v_{1}-V(t)\right) \leq 0} K\left(v_{1}-V(t) ; u_{1}-V(t)\right) f_{-}\left(t, \mathbf{x}, u_{1}, v_{\perp}\right) d u_{1}
$$

for $\mathbf{x} \in D(t)$, where the pre/postcollision distributions $f_{ \pm}$are defined as

$$
f_{ \pm}(t, \mathbf{x}, v)=\lim _{\varepsilon \rightarrow 0^{+}} f(t \pm \varepsilon, \mathbf{x} \pm \varepsilon \mathbf{v}, \mathbf{v}) .
$$

In (1.2) we regard $\mathbf{u}$ as the velocity of a particle coming into collision and $\mathbf{v}$ as an ejected velocity. We assume that the collision kernel $K$ conserves mass during collisions. The form of (1.2) implies that there is no energy exchange between the disk and the particles except in the horizontal direction. This is natural because the disk is fixed to move only along the $x$-axis. More detailed assumptions on $\mathbf{G}, f_{0}$ and $K$ are provided in Section 2. We note that the $F(t)$ notation for the frictional force does not reflect the complicated nature of the interaction. In fact, due to the recollisions between the body and the particles, we will see in Lemma 2.2 that the frictional force at time $t$ depends on the behavior of the whole system, including both the disk and the particles, at all previous times.

It is far from obvious what the ultimate velocity $V_{\infty}$ will be. We prove that $V_{\infty}$ is entirely determined by imagining a situation in which both $\mathbf{G}=\mathbf{0}$ and there are no precollisions; that is, the collisions at a time $t$ come from particles moving at constant velocities that have not previously collided with the body. The limiting velocity $V_{\infty}$ is determined as follows. We define a "fictitious" force $F_{00}$ for which there is no external field $\mathbf{G}$ at all and the particles do not hit the boundary before time $t$, namely,

$$
F_{00}(V)=\int_{D(t)} d S_{\mathbf{x}} \int_{\mathbb{R}^{3}} \tilde{L}\left(v_{1}-V(t)\right) f_{0}(\mathbf{v}) d \mathbf{v}
$$

where $\tilde{L}$ is defined in (2.8). It is a strictly monotone function of $V$. We then define $V_{\infty}$ by the equation

$$
F_{00}\left(V_{\infty}\right)=E .
$$

Our main result is stated informally as follows. If the initial velocity $V(0)$ is sufficiently close to $V_{\infty}$, then there exists a solution $(V(t), f(t, \mathbf{x}, \mathbf{v}))$ of our problem such that $V(t) \rightarrow V_{\infty}$ as $t \rightarrow \infty$. The same asymptotic statement is valid for any solution of the problem. This result is stated with precision and further details in Section 2. The present result is the first one for which the particles are themselves subject to a force $\mathbf{G} \neq \mathbf{0}$, so that between collisions with the body the individual particles travel in curves rather than straight lines. As a consequence, we have limited our investigation to proving the convergence (at some rate) to the equilibrium velocity $V_{\infty}$.

We state the basic iteration scheme to obtain the main theorem in (2.18). The core of the proof is a series of technical estimates. We emphasize that adding an external force on the continuum of particles, even considering only the rather ideal situation where the external force $\mathbf{G}(t, \mathbf{x})$ acting on them is small and decaying, makes the problem much more difficult to deal with. Because we are interested in the long-time behavior of the body, even a small force $\mathbf{G}$ could have significant effects for large time which we explain below.

Relating the aforementioned $V_{\infty}$, which comes from an imaginary case in which there are no collisions and $\mathbf{G}=\mathbf{0}$, to our system (for which there are collisions and $\mathbf{G} \neq \mathbf{0}$ ) is the most delicate and novel part 
of the analysis. In Section 3 we estimate the force $H(t)$ on the body at a large time $t$ due to $\mathbf{G}$ in the fictitious situation that there have been no precollisions. $H(t)$ is given by an integral over the various velocities $v$ of the incoming particles and we prove that $H(t)$ is integrable over $0<t<\infty$. We estimate the integral in five pieces, using an intermediate time $0<T<t$. One piece $H_{1}$ is small (for large $t$ ) because $v-V_{\infty}$ is small. The second piece $H_{21}$ is proven small by using the decay of $\mathbf{G}$ in time. The third piece $H_{221}$ makes use of the decay of $\mathbf{G}$ in both space and time. The fourth piece $H_{222}$ makes use of the decay of $\mathbf{G}$ in space. The fifth piece $H_{223}$ particularly makes use of the decay of $f_{0}$ as well as the decay of $\mathbf{G}$ in time. There is a range of possible choices for the parameters $p, m, q$, summarized in Theorem 3.2 .

Furthermore, because the body is moving and the particles travel in curves, it is evidently more difficult to track the collisions between the particles and the body, in contrast to the $\mathbf{G}=\mathbf{0}$ case for which the particles travel in straight lines. A given particle can collide with the body many times or even an infinite number of times. This is partially discussed in Lemma 2.2 and at the beginning of Section 4. Nevertheless, the effect of the precollisions can be estimated by the most recent precollision. This delicate part of the proof is given in Lemma 4.3. Finally, putting the different pieces together is a matter of dealing with several competing small quantities, as we discuss in Section 5, where we combine the previous estimates and prove the required convergence.

1.1. Acknowledgements. The authors would like to thank Kazuo Aoki once again for introducing them to this general subject and Yan Guo for discussions about multiple collisions. This research was supported in part by NSF grant DMS-1464869.

\section{Assumptions AND PRELIMINARIES}

We write the coordinates as $\mathbf{x}=\left(x_{1}, x_{\perp}\right)=\left(x_{1}, x_{2}, x_{3}\right), \mathbf{v}=\left(v_{1}, v_{\perp}\right)=\left(v_{1}, v_{2}, v_{3}\right), \mathbf{G}=\left(G_{1}, G_{\perp}\right)=$ $\left(G_{1}, G_{2}, G_{3}\right)$. We begin by listing the assumptions precisely. We assume that the force $\mathbf{G}$ has the form

$$
\mathbf{G}(t, \mathbf{x})=\left(G_{1}\left(t, x_{1}\right), G_{\perp}\left(t, x_{\perp}\right)\right)
$$

and that it decays like

$$
\left|G_{1}\left(t, x_{1}\right)\right| \leqslant \frac{c_{G}}{\langle t\rangle^{q}\left\langle x_{1}\right\rangle^{m}} \text { and }\left|G_{\perp}\left(t, x_{\perp}\right)\right| \leqslant \frac{c_{G}}{\langle t\rangle^{q}}
$$

where $c_{G}$ is a constant, $q>2$ and $m>0$. The structural assumption (2.1) is crucial to our proof although we hope to weaken it in future work. In the main theorem below we obtain a decay rate that does not depend on the dimension because we refrain from making an even stronger assumption. In fact, even with our decay assumption (2.2) on $G_{\perp}$, some of the particles might collide with the disk many times and might not even escape the disk at all.

We assume that the initial velocity distribution $f_{0}$ is a nonnegative even $C^{1}$ function $\not \equiv 0$ that has the form

$$
f_{0}(\mathbf{v})=a_{0}\left(v_{1}\right) b_{0}\left(v_{\perp}\right)
$$

and that it decays like

$$
\left|\nabla_{\mathbf{v}} f_{0}(\mathbf{v})\right| \leqslant \frac{C}{\left\langle v_{1}\right\rangle^{l_{1}+1}\left\langle v_{\perp}\right\rangle^{l_{2}+1}}
$$

where $l_{1}>q+1$ and $l_{2}>1$. (We remark that it is sufficient to assume the slightly weaker condition

$$
\int_{\mathbb{R}}\left|\frac{\partial a_{0}}{\partial v_{1}}\right|\left\langle v_{1}\right\rangle^{q} d v_{1}<\infty, \quad \int_{\mathbb{R}^{2}}\left|\nabla b_{0}\left(v_{\perp}\right)\right| d v_{\perp}<\infty
$$

although we will not bother to explicitly use this condition.)

Define $\gamma=\left|V(0)-V_{\infty}\right|$. This quantity is used throughout the paper and will be assumed to be very small. We assume that $K\left(v_{1}, u_{1}\right)$ is a continuous nonnegative function, $C^{1}$ for $u_{1} \neq 0, v_{1} \neq 0$, that is even in both variables separately $\left(K\left(v_{1}, u_{1}\right)=K\left(-v_{1}, u_{1}\right)=K\left(v_{1},-u_{1}\right)\right)$ and that is bounded for $v_{1} \in \mathbb{R},\left|u_{1}\right| \leq 3 \gamma$. We impose the mass conservation condition

$$
\int_{0}^{\infty} v_{1} K\left(v_{1}, u_{1}\right) d v_{1}=\left|u_{1}\right|
$$


the power law condition

$$
\sup _{\left|u_{1}\right|<\gamma+c_{G}} \int_{0}^{\infty} v_{1}^{2} K\left(v_{1}, u_{1}\right) d v_{1} \leq C\left|u_{1}\right|^{p}
$$

with $0<p \leq 2$, and the integrability condition

$$
K\left(v_{1}, z-y-V_{\infty}\right)\langle z\rangle^{-l_{1}} \leq M(z) \quad \text { for }\left|v_{1}\right|<3 \gamma,|y|<3 \gamma,|z|<\infty
$$

where $M \in L^{1}(\mathbb{R})$. We also define

$$
L\left(u_{1}\right)=u_{1}^{2}+\int_{\mathbb{R}} v_{1}^{2} K\left(v_{1}, u_{1}\right) d v_{1} \quad \text { and } \quad \tilde{L}\left(u_{1}\right)=\operatorname{sgn}\left(u_{1}\right) L\left(u_{1}\right) .
$$

Because $K$ is even, $L\left(u_{1}\right)$, as defined, is also even. In addition, we assume that the even function $L\left(u_{1}\right)$ is decreasing for $u_{1}<0$. Then we define the collision operator as $\mathcal{K}_{t}\left(f_{-}\right)=f_{+}$as in (1.2).

To summarize, the assumptions listed above have five exponents $p, m, q, l_{1}, l_{2}$ that satisfy $0<p \leq$ $2, q>2, m>0, l_{1}>q+1, l_{2}>1$. We define another parameter $\sigma$ by

$$
\frac{1}{\sigma}=\frac{1}{p+1}+\frac{1}{\mu}, \quad \mu=\min (m, q-1)
$$

We also have the two parameters $\gamma$ and $c_{G}$ in (2.2) that will be chosen sufficiently small. Our main theorem is as follows.

Theorem 2.1. Let $\mu>1+\frac{1}{p}$. There is a constant $A$ such that if $\gamma=\left|V(0)-V_{\infty}\right|$ and $c_{G}$ are sufficiently small, then there exists a solution $(V(t), f(t, \mathbf{x}, \mathbf{v}))$ of our problem for which $V \in C^{1}([0, \infty))$, $f \in L^{\infty}\left([0, \infty) \times \mathbb{R}^{3} \times \mathbb{R}^{3}\right)$ and

$$
\left|V(t)-V_{\infty}\right| \leqslant \gamma e^{-b_{0} t}+\frac{A \gamma^{p+1}}{(1+t)^{\sigma}}
$$

where $b_{0}=\min _{V \in\left[V_{\infty}-3 \gamma, V_{\infty}+3 \gamma\right]} F_{00}^{\prime}(V)$. The pair of functions $f_{ \pm}(t, \mathbf{x}, \mathbf{v})$ are a.e. defined explicitly in terms of $V(t)$ and $f_{0}(\mathbf{v})$. Furthermore, any solution of the problem (in the sense stated above) satisfies (2.10).

We now mention three examples of collision kernels for which the reflected velocity distribution is Gaussian for each incoming particle.

Example 1. Let

$$
K\left(v_{1}, u_{1}\right)=2 \beta e^{-\beta v_{1}^{2}}\left|u_{1}\right| .
$$

As shown in [9, 10], $K\left(v_{1}, u_{1}\right)$ satisfies the assumptions with $p=1$. In this case, we require $q>3$ and $m>2$ in (2.2), and $l_{1}>4$ in (2.4).

Example 2. We now choose

$$
K\left(v_{1}, u_{1}\right)=2 e^{-\frac{v_{1}^{2}}{\left|u_{1}\right|}} .
$$

It is proved in [9, 10] that this kernel checks our assumptions with $p=\frac{3}{2}$. In this case, we need $q>8 / 3$ and $m>5 / 3$ in (2.2), and $l_{1}>11 / 2$ in (2.4). In this example an incoming particle with almost the same velocity as the body is likely to be reflected with almost the same velocity, while an incoming particle with a very different velocity is reflected according to a very wide Gaussian around $V(t)$.

Example 3. We now scale the last example by setting

$$
K\left(v_{1}, u_{1}\right)=C\left|u_{1}\right|^{\beta} e^{-v_{1}^{2}\left|u_{1}\right|^{\beta-1}}
$$

where $\beta \in[-1,3)$ and $C$ is chosen such that mass is conserved, that is, (2.5) is satisfied. The most important feature of this kernel is that, as $\beta$ runs through $[-1,3)$, $p$ runs through $(0,2]$. At the endpoints of this interval we have the following behavior. If $p=2$, then $q>5 / 2$ and $m>3 / 2$ in (2.2), $l_{1}>7 / 2$ in 2.4). If $p \rightarrow 0$, then $q, m \rightarrow \infty$ in (2.2), $l_{1} \rightarrow \infty$ in 2.4).

We proceed by pointing out some basic properties of our model. The total mass $M=\int d \mathbf{x} \int d \mathbf{v} f(t, \mathbf{x}, \mathbf{v})$ of the particles is an invariant. Indeed, it is obviously invariant under the flow (1.1) while it is also preserved under collisions due to (2.5), as proven in [9] Lemma 2.3]. The total horizontal force at time $t$ due to the collisions is

$$
F(t)=\int_{D(t)} d S_{\mathbf{x}} \int_{\mathbb{R}^{3}} d \mathbf{v} \tilde{L}\left(v_{1}-V(t)\right) f_{-}(t, \mathbf{x}, \mathbf{v})
$$


as shown in [9, Lemma 2.2]. It is the sum of the force due to particles on the right $\left(v_{1}<V(t)\right)$ and the left $\left(v_{1}>V(t)\right)$.

Our basic technique is to look for possible velocities in the class $\mathcal{W}$, where we define $W \in \mathcal{W}$ if and only if $W(t)$ is Lipschitz, $W(0)=V_{0}$, and

$$
\left|W(t)-V_{\infty}\right| \leqslant \gamma e^{-b_{0} t}+\frac{A \gamma^{p+1}}{(1+t)^{\sigma}}
$$

where the constant $A$ will be specified later in Lemma 5.2 and the power $\sigma$ is given by (2.9). Among other conditions to be stated later, we require $A>1$ and $\gamma<<1$ so that $A \gamma<1$. For $W \in \mathcal{W}$ we define $X(t)$ as the primitive of $W(t)$ :

$$
\frac{d X}{d t}=W, \quad X(0)=0 .
$$

Until the last section of this paper we will fix any possible velocity $W \in \mathcal{W}$.

We introduce the characteristics of the PDE (1.1) without collisions as follows. Given $(t, \mathbf{x}, \mathbf{v})$, define $(\check{\mathbf{x}}(s ; t, \mathbf{x}, \mathbf{v}), \check{\mathbf{v}}(s ; t, \mathbf{x}, \mathbf{v}))$ to be the solution to the characteristic equations

$$
\begin{aligned}
\frac{d \check{\mathbf{x}}(s ; t, \mathbf{x}, \mathbf{v})}{d s} & =\check{\mathbf{v}}(s ; t, \mathbf{x}, \mathbf{v}) \\
\frac{d \check{\mathbf{v}}(s ; t, \mathbf{x}, \mathbf{v})}{d s} & =\mathbf{G}(s, \check{\mathbf{x}}(s ; t, \mathbf{x}, \mathbf{v}))
\end{aligned}
$$

with final condition $(\check{\mathbf{x}}(t ; t, \mathbf{x}, \mathbf{v}), \check{\mathbf{v}}(t ; t, \mathbf{x}, \mathbf{v}))=(\mathbf{x}, \mathbf{v})$. In particular, for $\mathbf{G}(t, \mathbf{x})$ of the separated form (2.1), the horizontal and vertical components are decoupled:

$$
\begin{aligned}
\left(\check{x}_{1}(r ; t, \mathbf{x}, \mathbf{v}), \check{v}_{1}(r ; t, \mathbf{x}, \mathbf{v})\right) & =\left(\check{x}_{1}\left(r ; t, x, v_{1}\right), \check{v}_{1}\left(r ; t, x, v_{1}\right)\right) \\
\left(\check{x}_{\perp}(r ; t, \mathbf{x}, \mathbf{v}), \check{v}_{\perp}(r ; t, \mathbf{x}, \mathbf{v})\right) & =\left(\check{x}_{\perp}\left(r ; t, x_{\perp}, v_{\perp}\right), \check{v}_{\perp}\left(r ; t, x_{\perp}, v_{\perp}\right)\right) .
\end{aligned}
$$

For any function $\phi$ on phase space, we define the solution operator $\mathcal{S}_{t r}$ as

$$
\left(\mathcal{S}_{t r} \phi\right)(\mathbf{x}, \mathbf{v})=\phi(\check{\mathbf{x}}(r ; t, \mathbf{x}, \mathbf{v}), \check{\mathbf{v}}(r ; t, \mathbf{x}, \mathbf{v})) .
$$

Then of course $f_{N B}=\mathcal{S}_{t 0} f_{0}$ solves the Vlasov equation

$$
\begin{aligned}
\left(\partial_{t}+\mathbf{v} \cdot \nabla_{\mathbf{x}}+\mathbf{G}(t, \mathbf{x}) \cdot \nabla_{\mathbf{v}}\right) f_{N B} & =0 \\
f_{N B}(0, \mathbf{x}, \mathbf{v}) & =f_{0}(\mathbf{v})
\end{aligned}
$$

in $\mathbb{R}^{3}$ with no boundary conditions.

The fictitious force $F_{00}$, previously defined in (1.3), is a strictly monotone function of $V$, due to $L$ being an even function that is decreasing for $u_{1}<0$. For completeness we state this as a lemma. As a consequence, $V_{\infty}$ will be uniquely defined by $F_{00}\left(V_{\infty}\right)=E$.

Lemma 2.1. Suppose $f_{0}(\mathbf{v}) \geqslant 0$ is even, continuous and $\not \equiv 0$. If $L \in C^{1}$ is an even function with $L(0)=0$, and $L^{\prime}\left(u_{1}\right)<0$ for $u_{1} \in(-\infty, 0)$, then $F_{00}(V)$ is an increasing odd $C^{1}$ function of $V$.

Proof. A simple change of variable yields

$$
\begin{aligned}
F_{00}(V) & =C\left(\int_{v_{1} \leqslant V} L\left(v_{1}-V\right) f_{0}(\mathbf{v}) d \mathbf{v}-\int_{v_{1} \geqslant V} L\left(v_{1}-V\right) f_{0}(\mathbf{v}) d \mathbf{v}\right) \\
& =C\left(\int_{v_{1} \leqslant V} L\left(v_{1}-V\right) f_{0}(\mathbf{v}) d \mathbf{v}-\int_{v_{1} \leqslant-V} L\left(v_{1}+V\right) f_{0}(\mathbf{v}) d \mathbf{v}\right)
\end{aligned}
$$

where $C$ is a fixed constant. Thus $F_{00}$ is odd and its derivative is

$$
F_{00}^{\prime}(V)=-C\left(\int_{v_{1} \leqslant V} L^{\prime}\left(v_{1}-V\right) f_{0}(\mathbf{v}) d \mathbf{v}+\int_{v_{x} \leqslant-V} L^{\prime}\left(v_{1}+V\right) f_{0}(\mathbf{v}) d \mathbf{v}\right)>0 .
$$

Given a time $t$, we will have to estimate the frictional force due to the collisions of the particles. To that end, we define another "fictitious" force as follows (though not as "fictitious" as $F_{00}$ ). The fictitious force $F_{0}(t)$ for which no particle hits the boundary before time $t$ is

$$
F_{0}(t)=F_{0}(t, W(t))=\int_{D(t)} d S_{\mathbf{x}} \int_{\mathbb{R}^{d}} \tilde{L}\left(v_{1}-W(t)\right) f_{N B}(t, \mathbf{x}, \mathbf{v}) d \mathbf{v}
$$


where $f_{N B}(t, \mathbf{x}, \mathbf{v})$ solves the kinetic equation (2.15) with no boundary condition and is subject to the initial condition $f_{N B}(0, \mathbf{x}, \mathbf{v})=f_{0}(\mathbf{v})$. As before, the fictitious force $F_{00}$ is defined so that there is no external field $\mathbf{G}$ at all and the particles do not hit the boundary before time $t$, namely,

$$
F_{00}=F_{00}(W(t))=\int_{D(t)} d S_{\mathbf{x}} \int_{\mathbb{R}^{d}} \tilde{L}\left(v_{1}-W(t)\right) f_{0}(\mathbf{v}) d \mathbf{v} .
$$

The force due to the precollisions on the body moving at velocity $W(t)$ then can be expressed as

$$
\begin{aligned}
R_{W}(t)= & F(t, W(t))-F_{0}(W(t)) \\
= & \int_{D(t)} \int_{v_{1} \geqslant W(t)} L\left(v_{1}-W(t)\right)\left[f_{N B}(t, \mathbf{x}, \mathbf{v})-f_{-}(t, \mathbf{x}, \mathbf{v})\right] d \mathbf{v} d S_{\mathbf{x}} \\
& +\int_{D(t)} \int_{v_{1} \leqslant W(t)} L\left(v_{1}-W(t)\right)\left[f_{-}(t, \mathbf{x}, \mathbf{v})-f_{N B}(t, \mathbf{x}, \mathbf{v})\right] d \mathbf{v} d S_{\mathbf{x}} \\
\equiv & R_{W}^{L}(t)+R_{W}^{R}(t)
\end{aligned}
$$

comprised of the forces on the right and left sides.

Given any $W \in \mathcal{W}$, we define the function $V_{W}(t)$ by the iteration scheme

$$
\frac{d V_{W}}{d t}=\frac{E-F_{00}(W)}{V_{\infty}-W}\left(V_{\infty}-V_{W}\right)-R_{W}(t)+F_{00}(W)-F_{0}(t, W), \quad V_{W}(0)=V_{0} .
$$

Note that a fixed point (that is, $V_{W}=W=V$ ) would satisfy

$$
\frac{d V}{d t}=\frac{E-F_{00}(V)}{V_{\infty}-V}\left(V_{\infty}-V\right)-R_{V}(t)+F_{00}(V)-F_{0}(t, V)=E-R_{V}(t)-F_{0}(t, V)
$$

and $d X / d t=V$. Thus any fixed point solves our problem. We will estimate the term $F_{00}(W)-F_{0}(t, W)$ in Section 3 and estimate the term $R_{W}(t)$ in Section 4 .

A key difficulty in our problem is that a typical particle may collide with the body many, or even infinitely many, times. The following lemma, written for convenience in the $1 \mathrm{D}$ case, illustrates this difficulty. It explains how one can represent the solution by an expansion in terms of a finite number $k$ of previous collisions. This expansion might never reach the initial datum if there are infinitely many collisions; it could happen in many ways.

Lemma 2.2 (Integral Representation). Let $(x, v) \notin Z(t)$ where $Z(t)$ is the set of measure zero defined in the beginning of Section 4 . For brevity we write $\mathcal{B}_{t r}=\mathcal{S}_{t r} \mathcal{K}_{t}$ and $J(s, x, v)=-G(s, x) f_{0}^{\prime}(v)$. Given $t>0$, we denote the particle collision times as $t>t_{1}>t_{2}>\ldots$. Then for arbitrary $k \geqslant 2$ we can represent the solution to (1.1) subject to the collision law (1.2) by

$$
\begin{aligned}
f(t)= & \sum_{j=1}^{k-1}\left\{\mathcal{B}_{t t_{1}} \ldots \mathcal{B}_{t_{j-1} t_{j}} \int_{\max \left(t_{j+1}, 0\right)}^{t_{j}} \mathcal{S}_{t_{j} s} J(s) d s\right\} \\
& +\chi_{\left\{0<t_{k}\right\}} \mathcal{B}_{t t_{1} \ldots \mathcal{B}_{t_{k-1}} t_{k} f_{-}\left(t_{k}\right)} \\
& +\int_{\max \left(t_{1}, 0\right)}^{t} \mathcal{S}_{t s} J(s) d s
\end{aligned}
$$

Proof. It is important to note that the actual number of precollisions could be larger than $k$ in formula (2.19). Thus if there are an infinite number of collisions, $f_{0}$ would never show up in (2.19). Note also that each $t_{j}$ depends on $t, x, v$ as well as on the pre/postcollision velocities at all $t_{i}$ for $1 \leqslant i<j$. Because the lemma is similar to [12, Lemma 24], we only sketch the proof here. It is instructive to first write out some of the terms in formula (2.19) in total detail including all the variables. For example, we have

$$
\mathcal{B}_{t t_{1}} g(x, v)=\mathcal{S}_{t, t_{1}} \mathcal{K} g(x, v)=\int d u_{1} K\left(\check{v}\left(t_{1}^{+} ; t, x, v\right)-V\left(t_{1}\right), u_{1}-V\left(t_{1}\right)\right) g\left(X\left(t_{1}\right), u_{1}\right)
$$

and

$$
\begin{aligned}
& \mathcal{B}_{t t_{1}} \mathcal{B}_{t_{1} t_{2}} g(x, v)=\int d u_{1} K\left(\check{v}\left(t_{1}^{+} ; t, x, v\right)-V\left(t_{1}\right), u_{1}-V\left(t_{1}\right)\right) \\
& \times \int d u_{2} K\left(\check{v}\left(t_{2}^{+} ; t_{1}, X\left(t_{1}\right), u_{1}\right)-V\left(t_{2}\right), u_{2}-V\left(t_{2}\right)\right) g\left(X\left(t_{2}\right), u_{2}\right)
\end{aligned}
$$


where $t_{i}^{+}$means $\lim _{t \rightarrow t_{i}^{+}}$, that is, postcollision. In fact, exhibiting all the variables, we have

$$
\begin{aligned}
& \mathcal{B}_{t t_{1}} \ldots \mathcal{B}_{t_{j-1} t_{j}} g(x, v)=\int d u_{1} K\left(\check{v}\left(t_{1}^{+} ; t, x, v\right)-V\left(t_{1}\right), u_{1}-V\left(t_{1}\right)\right) \times \ldots \\
& \times \int d u_{j} K\left(\check{v}\left(t_{j}^{+} ; t_{j-1}, X\left(t_{j-1}\right), u_{j-1}\right)-V\left(t_{j}\right), u_{j}-V\left(t_{j}\right)\right) g\left(X\left(t_{j}\right), u_{j}\right) .
\end{aligned}
$$

We now begin the formal proof with the standard Duhamel formula

$$
f(t)=\chi_{\left\{0<t_{1}\right\}} \mathcal{S}_{t t_{1}} \mathcal{K}_{t_{1}} f_{-}\left(t_{1}\right)+\int_{\max \left(t_{1}, 0\right)}^{t} \mathcal{S}_{t s} J(s) d s .
$$

We then write the next Duhamel formula

$$
f_{-}\left(t_{1}\right)=\chi_{\left\{0<t_{2}\right\}} \mathcal{S}_{t_{1} t_{2}} \mathcal{K}_{t_{2}} f_{-}\left(t_{2}\right)+\int_{\max \left(t_{2}, 0\right)}^{t_{1}} \mathcal{S}_{t_{1} s} J(s) d s .
$$

Combining the two formulas, we obtain

$$
f(t)=\chi_{\left\{0<t_{2}\right\}} \mathcal{B}_{t t_{1}} \mathcal{B}_{t_{1} t_{2}} f_{-}\left(t_{2}\right)+\mathcal{B}_{t t_{1}} \int_{\max \left(t_{2}, 0\right)}^{t_{1}} \mathcal{S}_{t_{1} s} J(s) d s+\int_{\max \left(t_{1}, 0\right)}^{t} \mathcal{S}_{t s} J(s) d s .
$$

This is the case $k=2$. Iterating in the same manner by induction, that is, replacing $f_{-}\left(t_{j}\right)$ with its expression at the next level in order to get $f_{-}\left(t_{j+1}\right)$, we obtain formula (2.19).

\section{EFFECT OF THE PARTICLE FORCES ON THE BODY}

The difference between the two fictitious forces (2.16) and (2.17) is

$$
H(t)=H(V(t))=F_{00}(t)-F_{0}(t)=\int_{D(t)} d S_{\mathbf{x}} \int_{\mathbb{R}^{d}} \tilde{L}\left(v_{1}-V(t)\right) h(t, \mathbf{x}, \mathbf{v}) d \mathbf{v}
$$

where we denote

$$
h(t, \mathbf{x}, \mathbf{v})=f_{N B}(t, \mathbf{x}, \mathbf{v})-f_{0}(\mathbf{v}) .
$$

In this expression there is no collision with the body. The function $h$ solves

$$
\begin{aligned}
\left(\partial_{t}+\mathbf{v} \cdot \nabla_{\mathbf{x}}+\mathbf{G}(t, \mathbf{x}) \cdot \nabla_{\mathbf{v}}\right) h & =-\mathbf{G}(t, \mathbf{x}) \cdot \nabla_{\mathbf{v}} f_{0} \\
h(0, \mathbf{x}, \mathbf{v}) & =0
\end{aligned}
$$

or written in Duhamel form,

where we also denote

$$
h(t, \mathbf{x}, \mathbf{v})=\int_{0}^{t} \mathcal{S}_{t s} J(s) d s
$$

$$
J(s)=J(s, \mathbf{x}, \mathbf{v})=-\mathbf{G}(s, \mathbf{x}) \cdot \nabla_{\mathbf{v}} f_{0} .
$$

For any $T \in[0, t]$, we could also write

$$
h(t, \mathbf{x}, \mathbf{v})=h(T, \check{\mathbf{x}}(T ; t, \mathbf{x}, \mathbf{v}), \check{\mathbf{v}}(T ; t, \mathbf{x}, \mathbf{v}))-\int_{T}^{t} \mathbf{G}(s, \check{\mathbf{x}}(s ; t, \mathbf{x}, \mathbf{v})) \cdot \nabla_{\mathbf{v}} f_{0}(\check{\mathbf{v}}(s ; t, \mathbf{x}, \mathbf{v})) d s .
$$

We claim that $H(t)$ is small and integrable in time so long as $\mathbf{G}$ is small. To prove such a claim, we first need the following lemma. The constants denoted as $C$ occurring in the estimates throughout this paper are independent of $\gamma, c_{G}, t$, and $A$ as well as the solution. The constant $A$ will be chosen sufficiently large relative to one of the constants $C$.

Lemma 3.1. There is a constant $C>0$ independent of $c_{G}, \gamma, t, \mathbf{x}$, and $\mathbf{v}$, such that

$$
|h(t, \mathbf{x}, \mathbf{v})| \leqslant c_{G} C \int_{0}^{t} \frac{1}{\langle s\rangle^{q}} \frac{1}{\left\langle\check{x}_{1}(s ; t, \mathbf{x}, \mathbf{v})\right\rangle^{m}} d s \frac{1}{\left\langle v_{1}\right\rangle^{l_{1}+1}} \frac{1}{\left\langle v_{\perp}\right\rangle^{l_{2}+1}} \leq c_{G} C .
$$

Proof. We have

$$
\mathcal{S}_{t s} J(s)=-\mathbf{G}(s, \check{\mathbf{x}}(s ; t, \mathbf{x}, \mathbf{v})) \cdot\left(\nabla_{\mathbf{v}} f_{0}\right)(\check{\mathbf{v}}(s ; t, \mathbf{x}, \mathbf{v})) .
$$

So by assumption,

$$
\left|\mathcal{S}_{t s} J(s)\right| \leqslant c_{G} C \frac{1}{\langle s\rangle^{q}} \frac{1}{\left\langle\check{x}_{1}(s ; t, \mathbf{x}, \mathbf{v})\right\rangle^{m}} \frac{1}{\left\langle\check{v}_{1}(s ; t, \mathbf{x}, \mathbf{v})\right\rangle^{l_{1}+1}} \frac{1}{\left\langle\check{v}_{\perp}(s ; t, \mathbf{x}, \mathbf{v})\right\rangle^{l_{2}+1}} .
$$


Hence

$$
\begin{aligned}
& |h(t, \mathbf{x}, \mathbf{v})| \leqslant \int_{0}^{t}\left|\mathcal{S}_{t s} J(s)\right| d s \\
\leqslant & c_{G} C \int_{0}^{t} \frac{1}{\langle s\rangle^{q}} \frac{1}{\left\langle\check{x}_{1}(s ; t, \mathbf{x}, \mathbf{v})\right\rangle^{m}} \frac{1}{\left\langle\check{v}_{1}(s ; t, \mathbf{x}, \mathbf{v})\right\rangle^{l_{1}+1}} \frac{1}{\left\langle\check{v}_{\perp}(s ; t, \mathbf{x}, \mathbf{v})\right\rangle^{l_{2}+1}} d s .
\end{aligned}
$$

Finally notice that

$$
\begin{aligned}
\left|\check{v}_{1}(s ; t, \mathbf{x}, \mathbf{v})-v_{1}\right| & \leqslant \int|\mathbf{G}(p, \check{\mathbf{x}}(p ; t, \mathbf{x}, \mathbf{v}))| d p \leqslant 2 \int \frac{c_{G}}{\langle p\rangle^{q}} d p \leqslant c_{G} C \\
\left|\check{v}_{\perp}(s ; t, \mathbf{x}, \mathbf{v})-v_{\perp}\right| & \leqslant \int|\mathbf{G}(p, \check{\mathbf{x}}(p ; t, \mathbf{x}, \mathbf{v}))| d p \leqslant 2 \int \frac{c_{G}}{\langle p\rangle^{q}} d p \leqslant c_{G} C .
\end{aligned}
$$

So we can replace $\check{v}_{1}(s ; t, \mathbf{x}, \mathbf{v})$ and $\check{v}_{\perp}(s ; t, \mathbf{x}, \mathbf{v})$ in estimate (3.3) by $v_{1}$ and $v_{\perp}$ at the price of a different constant $C$. Thereby we obtain estimate (3.2).

The next two lemmas provide rather delicate estimates of $H(t)$.

Lemma 3.2. We select $T=t^{\alpha}$ with $0<\alpha<1$ to be determined later and assume $0<p \leq 2$ and the following (somewhat redundant) decay conditions:

$$
\begin{gathered}
(1-\alpha)(p+1)>1 \\
l_{1}>2 \text { and } \alpha(q-1)>1 \\
\alpha m>1, q>2 \\
l_{1}>q+1 \text { and } \alpha(q-1)>1 .
\end{gathered}
$$

Then the two fictitious forces $F_{0}$ and $F_{00}$ are near each other in the sense that there is $C$ independent of $t$ such that

$$
|H(t)| \leqslant c_{G} C\left[\frac{1}{\langle t\rangle^{(1-\alpha)(p+1)}}+\frac{1}{\langle t\rangle^{\alpha m}}+\frac{1}{\langle t\rangle^{\alpha(q-1)}}\right] .
$$

Proof. Recall

$$
H(t)=\int_{D(t)} d S_{\mathbf{x}} \int_{\mathbb{R}^{d}} \tilde{L}\left(v_{1}-W(t)\right) h(t, \mathbf{x}, \mathbf{v}) d \mathbf{v} .
$$

First we consider $t \leq 1$. Note from (2.8) that

$$
\tilde{L}\left(v_{1}-W(t)\right) \leq C\left(\left|v_{1}-W(t)\right|^{p}+\left|v_{1}-W(t)\right|^{2}\right) \leq C\left\langle v_{1}\right\rangle^{2}
$$

because $p \leq 2$ and

$$
\left|v_{1}-W(t)\right| \leq\left|v_{1}-V_{\infty}\right|+\left|V_{\infty}-W(t)\right| \leqslant\left|v_{1}\right|+\left|V_{\infty}\right|+(\gamma+A \gamma) \leqslant C\left\langle v_{1}\right\rangle
$$

for $t \leq 1$. In the previous estimates we have used the assumption that $A \gamma \leqslant 1$ and $\gamma$ is small. By Lemma 3.1 we therefore have

$$
|H(t)| \leq \int_{D(t)} d S_{\mathbf{x}} \int\left\langle v_{1}\right\rangle^{2} C c_{G}\left\langle v_{1}\right\rangle^{-l_{1}-1}\left\langle v_{\perp}\right\rangle^{-l_{2}-1} d \mathbf{v} \leq C c_{G}
$$

because $l_{1}>2$ and $l_{2}>1$.

In the rest of the proof, we assume $t>1$. We decompose $H(t)=H_{1}(t)+H_{2}(t)$, where

$$
\begin{aligned}
H_{1}(t) & =\int_{D(t)} d S_{\mathbf{x}} \int_{S} \tilde{L}\left(v_{1}-W(t)\right) h(t, \mathbf{x}, \mathbf{v}) d \mathbf{v} \\
H_{2}(t) & =\int_{D(t)} d S_{\mathbf{x}} \int_{S^{C}} \tilde{L}\left(v_{1}-W(t)\right) h(t, \mathbf{x}, \mathbf{v}) d \mathbf{v}
\end{aligned}
$$

and

$$
S=\left\{\mathbf{v} \in \mathbb{R}^{d}:\left|v_{1}-V_{\infty}\right| \leqslant \frac{b T}{t}\right\} .
$$

The constant $b$ will be specified later and we choose $T=t^{\alpha}$. 
The estimate for $H_{1}$ is fairly simple. In fact, we have by Lemma 3.1 that

$$
\begin{aligned}
\left|H_{1}(t)\right| & \leqslant c_{G} C \int_{D(t)} d S_{\mathbf{x}} \int_{\left|v_{1}-V_{\infty}\right| \leqslant \frac{b T}{t}} d v_{1} \tilde{L}\left(v_{1}-W(t)\right) \int d v_{\perp} \frac{1}{\left\langle v_{\perp}\right\rangle^{l_{2}+1}} \\
& \leqslant c_{G} C \int_{\left|v_{1}-V_{\infty}\right| \leq \frac{b T}{t}}\left\{\left|v_{1}-V_{\infty}\right|^{p}+\left|v_{1}-V_{\infty}\right|^{2}+\left|V_{\infty}-W(t)\right|^{p}+\left|V_{\infty}-W(t)\right|^{2}\right\} d v_{1} \\
& \leqslant c_{G} C b^{p+1}\left(\frac{T}{t}\right)^{p+1}+c_{G} C \frac{1}{t^{p \sigma}} b \frac{T}{t}
\end{aligned}
$$

where the last inequality comes from $p \leq 2$ and the definition of $\mathcal{W}$. Note that no extra decay can be obtained from the vertical component involving $v_{\perp}$. Now $\min (m, q-1)>\frac{1}{\alpha}$ due to (3.6) and (3.7), so that by (3.8) we have $\sigma>1>1-\alpha$ and

$$
(p+1)(1-\alpha)<p \sigma+1-\alpha, \text { for } p>0
$$

that is, the second term can be absorbed into the first one:

$$
\left|H_{1}(t)\right| \leqslant c_{G} C\left(\frac{1}{t^{(1-\alpha)(p+1)}}+\frac{1}{t^{p \sigma+(1-\alpha)}}\right) \leq c_{G} C \frac{1}{t^{(1-\alpha)(p+1)}}
$$

which is integrable in $t$ due to (3.5).

We now turn our focus to $H_{2}(t)$, which is split into two parts $H_{2}=H_{21}+H_{22}$ as follows.

$$
\begin{aligned}
H_{21}(t) & =\int_{D(t)} d S_{\mathbf{x}} \int_{S^{C}} \tilde{L}\left(v_{1}-W(t)\right)\left[\int_{T}^{t} \mathcal{S}_{t s} J(s) d s\right] d \mathbf{v} \\
H_{22}(t) & =\int_{D(t)} d S_{\mathbf{x}} \int_{S^{C}} \tilde{L}\left(v_{1}-W(t)\right) h(T, \check{\mathbf{x}}(T ; t, \mathbf{x}, \mathbf{v}), \check{\mathbf{v}}(T ; t, \mathbf{x}, \mathbf{v})) d \mathbf{v}
\end{aligned}
$$

where $h(T, \check{\mathbf{x}}(T ; t, \mathbf{x}, \mathbf{v}), \check{\mathbf{v}}(T ; t, \mathbf{x}, \mathbf{v}))=\int_{0}^{T} \mathcal{S}_{t s} J(s) d s$. Now

$$
\begin{aligned}
\left|H_{21}(t)\right| & \leqslant c_{G} C \int_{D(t)} d S_{\mathbf{x}} \int_{S^{C}} \int_{T}^{t} \tilde{L}\left(v_{1}-W(t)\right) \frac{1}{\langle s\rangle^{q}} \frac{1}{\left\langle v_{1}\right\rangle^{l_{1}+1}} \frac{1}{\left\langle v_{\perp}\right\rangle^{l_{2}+1}} d s d \mathbf{v} \\
& \leqslant c_{G} C \int_{T}^{t} \frac{1}{s^{q}} \int_{S^{C}} C\left(\left|v_{1}-W(t)\right|^{2}+\left|v_{1}-W(t)\right|^{p}\right) \frac{1}{\left\langle v_{1}\right\rangle^{l_{1}+1}} \frac{1}{\left\langle v_{\perp}\right\rangle^{l_{2}+1}} d \mathbf{v} d s \\
& \leqslant c_{G} C \int_{T}^{t} \frac{1}{\langle s\rangle^{q}} d s \leqslant c_{G} C \frac{1}{T^{q-1}}=c_{G} C \frac{1}{t^{\alpha(q-1)}}
\end{aligned}
$$

which is integrable over $t \in(0, \infty)$ if (3.6) is satisfied. We note that the constant $C$ in $H_{21}$ depends on $V_{\infty}$. It remains to estimate $H_{22}$, which we put into Lemma 3.3 .

Lemma 3.3. Assuming the decay conditions (3.7) and (3.8), we have

$$
\left|H_{22}(t)\right| \leqslant c_{G} C\left[\frac{1}{\langle t\rangle^{\alpha m}}+\frac{1}{\langle t\rangle^{\alpha(q-1)}}\right] \text { and so } \int_{0}^{\infty}\left|H_{22}(t)\right| d t<\infty .
$$

Proof. Because the $t \leqslant 1$ case has already been taken care of in Lemma 3.2, we restrict ourselves to $t>1$ in this proof. Recall that we have chosen $T=t^{\alpha}<t$. For brevity we denote

$$
\check{\mathbf{x}}=\check{\mathbf{x}}(T ; t, \mathbf{x}, \mathbf{v}), \quad \check{\mathbf{v}}=\check{\mathbf{v}}(T ; t, \mathbf{x}, \mathbf{v}),
$$

and

$$
\mathbf{x}^{*}=\check{\mathbf{x}}(s ; T, \check{\mathbf{x}}, \check{\mathbf{v}})=\check{\mathbf{x}}(s ; t, \mathbf{x}, \mathbf{v}), \quad \mathbf{v}^{*}=\check{\mathbf{v}}(s ; T, \check{\mathbf{x}}, \check{\mathbf{v}}) .=\check{\mathbf{v}}(s ; t, \mathbf{x}, \mathbf{v}) .
$$

By Lemma 3.1 with $(t, \mathbf{x}, \mathbf{v})$ replaced by $(T, \check{\mathbf{x}}, \check{\mathbf{v}})$, we have

$$
\begin{aligned}
\left|H_{22}(t)\right| & \leqslant c_{G} C \int_{D(t)} d S_{\mathbf{x}} \int_{S^{C}} d \mathbf{v} \int_{0}^{T} d s \tilde{L}\left(v_{1}-W(t)\right) \frac{1}{\langle s\rangle^{q}} \frac{1}{\left\langle x_{1}^{*}\right\rangle^{m}} \frac{1}{\left\langle v_{1}\right\rangle^{l_{1}+1}} \frac{1}{\left\langle v_{\perp}\right\rangle^{l_{2}+1}} \\
& \leqslant c_{G} C \int_{D(t)} d S_{\mathbf{x}} \int_{S^{C}} d v_{1} \int_{0}^{T} d s \tilde{L}\left(v_{1}-W(t)\right) \frac{1}{\langle s\rangle^{q}} \frac{1}{\left\langle x_{1}^{*}\right\rangle^{m}} \frac{1}{\left\langle v_{1}\right\rangle^{l_{1}+1}} .
\end{aligned}
$$

We split $H_{22}$ into three parts $H_{22}=H_{221}+H_{222}+H_{223}$, where $H_{221}$ is the integral over the set

$$
\left\{\left|v_{1}\right|<1\right\} \cap S^{c}
$$


$H_{222}$ is the integral over

$$
\left\{\left|x_{1}^{*}\right| \geq(t / 2)\left|v_{1}-V_{\infty}\right|>b T / 2\right\} \cap\left\{\left|v_{1}\right| \geq 1\right\},
$$

and $H_{223}$ is the integral over

$$
\left\{\left|x_{1}^{*}\right|<(t / 2)\left|v_{1}-V_{\infty}\right|\right\} \cap\left\{\left|v_{1}\right| \geq 1\right\} \cap S^{c} .
$$

We begin with $H_{221}$. Solving the characteristic equations (2.13) for $x_{1}^{*}$ with final data at time $T$ using (2.2) and (3.12), we have

$$
\left|x_{1}^{*}\right| \geq\left|\check{x}_{1}-T \check{v}_{1}+s \check{v}_{1}\right|-c_{2}(T-s) \geq\left|\check{x}_{1}-T v_{1}\right|-T\left|\check{v}_{1}-v_{1}\right|-s\left|\check{v}_{1}\right|-c_{2}(T-s)
$$

where $c_{2}=O\left(c_{G}\right)$. We estimate two of these terms as follows. On the one hand, considering the time interval $(T, t)$ and using the characteristic equation (2.13), we have

$$
T\left|\check{v}_{1}-v_{1}\right| \leqslant C T \int_{T}^{t} \frac{1}{\langle p\rangle^{q}} d p \leq \frac{1}{\langle T\rangle^{q-2}} \leqslant C
$$

since $q>2$. On the other hand, for $t>1$ we have

$$
\begin{aligned}
\left|\check{x}_{1}-T v_{1}\right| & \geq t\left|V_{\infty}-v_{1}\right|-\left|\check{x}_{1}-X(t)-(T-t) v_{1}\right|-\left|X(t)-t V_{\infty}\right| \\
& \geq b T-\int_{T}^{t} \int_{T}^{\tau} \frac{1}{\langle p\rangle^{q}} d p d \tau-\int_{0}^{t}\left|W(\tau)-V_{\infty}\right| d \tau \\
& \geq b T-C \frac{t}{\langle T\rangle^{q-1}}-C(\gamma+A \gamma) \\
& \geq b T-C-C \frac{t}{\langle T\rangle^{q-1}} \geqslant b T-C
\end{aligned}
$$

for some constant $C$, where we used the fact that

$$
\int_{T}^{t}\left(\int_{T}^{\tau} \frac{1}{\langle p\rangle^{q}} d p\right) d \tau=C_{q} \int_{T}^{t}\left(\frac{1}{\langle T\rangle^{q-1}}-\frac{1}{\langle\tau\rangle^{q-1}}\right) d \tau \leqslant C_{q} \int_{T}^{t} \frac{d \tau}{\langle T\rangle^{q-1}}=C_{q} \frac{t-T}{\langle T\rangle^{q-1}} \leqslant C_{q} t^{1-\alpha(q-1)} \leqslant C
$$

since $q>2$ and $\alpha(q-1)>1$. Therefore, combining (3.14) with the two previous inequalities and using $\left|\breve{v}_{1}\right| \leq 1+c_{2}$, we have

$$
\left|x_{1}^{*}\right| \geq b T-C-c_{2} T-\left|\check{v}_{1}\right| s+c_{2} s \geq\left(2 c_{2}+2\right) T-C-\left(1+c_{2}\right) T \geq T-C
$$

for $0 \leq s \leq T=t^{\alpha}$ if we choose $b>3 c_{2}+2$. Plugging the lower bound of $\left|x_{1}^{*}\right|$ given above into (3.13), we have

$$
\begin{aligned}
\left|H_{221}(t)\right| & \leqslant c_{G} C \iint_{0}^{T} \frac{1}{\langle s\rangle^{q}} \frac{\left(\left|v_{1}-W(t)\right|^{2}+\left|v_{1}-W(t)\right|^{p}\right)}{\langle T\rangle^{m}\left\langle v_{1}\right\rangle^{l_{1}+1}} d s d v_{1} \\
& \leqslant c_{G} C \frac{1}{\langle T\rangle^{m}}=c_{G} C \frac{1}{\langle t\rangle^{\alpha m}} .
\end{aligned}
$$

Since $\alpha m>1,\left|H_{221}(t)\right|$ is integrable in $t$, as desired.

Now for $H_{222}$, we have, by definition, that $\left|x_{1}^{*}\right|>b T / 2$. Hence

$$
\left|H_{222}(t)\right| \leqslant c_{G} C \int \frac{1}{\langle s\rangle^{q}} d s \frac{\left(\left|v_{1}-W(t)\right|^{2}+\left|v_{1}-W(t)\right|^{p}\right)}{\langle T\rangle^{m}\left\langle v_{1}\right\rangle^{l_{1}+1}} d v_{1} \leqslant c_{G} C \frac{1}{\langle T\rangle^{m}}=c_{G} C \frac{1}{\langle t\rangle^{\alpha m}}
$$

which is also integrable in $t$.

Finally considering the term $H_{223}$ and using the characteristic equation (2.13) in the interval $(s, T)$ as before, we have

$\left|x_{1}^{*}\right| \geq\left|\check{x}_{1}-T \check{v}_{1}+s \check{v}_{1}\right|-c_{2}(T-s) \geq t\left|V_{\infty}-v_{1}\right|-\left|\check{x}_{1}-X(t)-(T-t) v_{1}\right|-\left|X(t)-t V_{\infty}\right|-s\left|\check{v}_{1}\right|-c_{2} T+c_{2} s$ where again $c_{2}=O\left(c_{G}\right)$. Consideration of the interval $(T, t)$ provides a constant bound of the second and third terms on the right side of this inequality. In fact, we already proved in (3.15) that

$$
\left|\check{x}_{1}-X(t)-(T-t) v_{1}\right|+\left|X(t)-t V_{\infty}\right| \leqslant C .
$$

Within the integration region $\left\{\frac{t}{2}\left|v_{1}-V_{\infty}\right|>\left|x_{1}^{*}\right|\right\}$ of $H_{223}$, we therefore have

$$
\frac{t}{2}\left|V_{\infty}-v_{1}\right|>\left|x_{1}^{*}\right| \geqslant t\left|V_{\infty}-v_{1}\right|-C-c_{2} T-s\left(\left|\check{v}_{1}\right|-c_{2}\right)
$$


where $C$ is some fixed constant. Hence

$$
s \geq \frac{1}{\left|\check{v}_{1}\right|-c_{2}}\left\{\frac{t}{2}\left|V_{\infty}-v_{1}\right|-C-c_{2} T\right\} \geq \frac{1}{\left|\check{v}_{1}\right|-c_{2}}\left\{\left(\frac{b}{4}-c_{2}\right) T-C\right\} \geq \frac{c_{5} T}{\left|\check{v}_{1}\right|-c_{2}} \equiv s_{0},
$$

where we have used the definition of $S^{c}$ and where $c_{5}>0$ provided that we choose $b$ large enough that $b>4 C+4 c_{2}$. Moreover, choosing $c_{G}$ small enough, we have by (3.4) that

$$
\left|\check{v}_{1}\right|-c_{2} \geqslant\left|v_{1}\right|-\left|v_{1}-\check{v}_{1}\right|-c_{2} \geqslant 1-C c_{G}-c_{2}>\frac{1}{2} .
$$

Thus from (3.13) we have

$$
\begin{aligned}
\left|H_{223}(t)\right| & \leq c_{G} C \int_{s_{0}}^{\infty} \frac{1}{\langle s\rangle^{q}} d s \int_{0}^{\infty} \frac{\left(\left|v_{1}-W(t)\right|^{2}+\left|v_{1}-W(t)\right|^{p}\right)}{\left\langle v_{1}\right\rangle^{l_{1}+1}} d v_{1} \\
& \leqslant \frac{c_{G} C}{T^{q-1}} \int_{0}^{\infty} \frac{\left\langle v_{1}\right\rangle^{q-1}}{\left\langle v_{1}\right\rangle^{l_{1}+1}}\left(\left|v_{1}-W(t)\right|^{2}+\left|v_{1}-W(t)\right|^{p}\right) d v_{1} .
\end{aligned}
$$

Thus

$$
\left|H_{223}(t)\right| \leqslant \frac{c_{G} C}{\langle T\rangle^{q-1}}=\frac{c_{G} C}{\langle t\rangle^{\alpha(q-1)}}
$$

because $l_{1}>q+1$. It is an integrable function of $t$ because $\alpha(q-1)>1$.

We summarize the estimates of $H(t)$ in the following theorem.

Theorem 3.1. Assuming $\mu \equiv \min (m, q-1)>\frac{p+1}{p}$, we have $|H(t)| \leqslant c_{G} C\langle t\rangle^{-\sigma}$, where $\sigma$ is given by (2.9).

Proof. By Lemma 3.2, we have

$$
\begin{aligned}
|H(t)| & \leqslant c_{G} C\left[\frac{1}{\langle t\rangle^{(1-\alpha)(p+1)}}+\frac{1}{\langle t\rangle^{\alpha m}}+\frac{1}{\langle t\rangle^{\alpha(q-1)}}\right] \\
& \leqslant c_{G} C\left[\frac{1}{\langle t\rangle^{(1-\alpha)(p+1)}}+\frac{1}{\langle t\rangle^{\alpha \min (m, q-1)}}\right]
\end{aligned}
$$

The inequality is optimized by equating the two powers. That is, we choose $\alpha=(p+1) /(p+1+\min (m, q-1))$. Then we get the stated inequality. If $\min (m, q-1)>\frac{p+1}{p}$, we automatically have

$$
\alpha \min (m, q-1)>1, \quad(1-\alpha)(p+1)>1 .
$$

\section{EFFECT OF THE PRECOLLISIONS ON THE BODY}

Because we have already estimated the term $F_{00}(W)-F_{0}(t, X, W)$ in Theorem 3.1, we may now focus on the estimate of $R_{W}(t)$.

We begin with a couple of observations. First, recall from the earlier discussion in Lemma 2.2 that a particle with position $\mathbf{x}$ and velocity $\mathbf{v}$ at time $t$ may have collided with the body at various earlier times $t>t_{1}>t_{2}>\ldots$ Given a particle colliding at time $t$, we define the infinite collision set $Z(t)$ to be comprised of all points $(\mathbf{x}, \mathbf{v})$ for which the particle also collides with the body at a sequence of times $s_{j} \rightarrow t$. If $v_{1}(s)$ denotes the horizontal velocity of such a particle at time $s$, then

$$
\int_{s_{j}}^{t} W(s) d s=\int_{s_{j}}^{t} v_{1}(s) d s
$$

for the sequence of times $s_{j} \rightarrow t$, so that $W(t)=v_{1}(t)=v_{1}$. Thus such particles comprise a set of measure zero and so provide no contribution to the force so that we may neglect them. For each of the remaining particles the collision time $t$ is isolated.

Secondly, away from $Z(t)$, each particle that collides at time $t$ either has a last precollision time $\tau(t, x, v)<t$ or else has no precollision at all in the interval $(0, t)$. Accordingly, we can write the particle density at time $t$ as the sum

$$
f_{-}(t, \mathbf{x}, v)=\chi_{1}(t, \mathbf{v}) f_{+}(\tau, \check{\mathbf{x}}(\tau ; t, \mathbf{x}, \mathbf{v}), \check{v}(\tau ; t, \mathbf{x}, \mathbf{v}))+\chi_{0}(t, \mathbf{v}) f_{0}(\check{v}(0 ; t, \mathbf{x}, \mathbf{v}))
$$


where the precollision characteristic functions $\chi_{1}(t, \mathbf{v})$ and $\chi_{0}(t, \mathbf{v})$ are defined as follows. $\chi_{1}(t, \mathbf{v})$ is the characteristic function of

$$
\left\{(t, \mathbf{v}) \mid \exists(\tau, \mathbf{x}) \in(0, t) \times D(t) \text { s.t. } \int_{\tau}^{t} W(s) d s=\int_{\tau}^{t} \check{v}_{x}(s ; t, \mathbf{x}, \mathbf{v}) d s, \int_{\tau}^{t}\left|\check{v}_{\perp}(s ; t, \mathbf{x}, \mathbf{v})\right| d s \leqslant 2 R\right\}
$$

with $R$ being the radius of the disk. Moreover, $\chi_{0}(t, \mathbf{v})=1-\chi_{1}(t, \mathbf{v})$. The following lemma provides a crude estimate on the velocity.

Lemma 4.1. If $\chi_{1}(t, \mathbf{v}) \neq 0$, then

$$
\inf _{s \leqslant t}\langle W\rangle_{s, t}-\frac{c_{G} C}{\langle t\rangle} \leqslant v_{1} \leqslant \sup _{s \leqslant t}\langle W\rangle_{s, t}+\frac{c_{G} C}{\langle t\rangle}
$$

where $\langle f\rangle_{s, t}$ denotes the average of $f$ over the interval $[s, t]$.

Proof. Taking the last collision time $\tau=\tau(t, \mathbf{x}, \mathbf{v})$ before $t$, we have from (2.13)

$$
(t-\tau) v_{1}-c_{G} \int_{\tau}^{t} \int_{s}^{t} \frac{1}{\langle p\rangle^{q}} d p d s \leqslant \int_{\tau}^{t} \check{v}_{1}(s ; t, \mathbf{x}, \mathbf{v}) d s \leqslant(t-\tau) v_{1}+c_{G} \int_{\tau}^{t} \int_{s}^{t} \frac{1}{\langle p\rangle^{q}} d p d s
$$

Now

$$
0 \leqslant \int_{\tau}^{t} \int_{s}^{t} \frac{1}{\langle p\rangle^{q}} d p d s \leqslant C \int_{\tau}^{t} \frac{d s}{\langle s\rangle^{q-1}}-\frac{d s}{\langle t\rangle^{q-1}} \leqslant C \int_{\tau}^{t} \frac{d s}{\langle s\rangle^{q-1}}=C(t-\tau)\left\langle\langle s\rangle^{-q+1}\right\rangle_{\tau, t}
$$

with $C$ depending on $q$. Hence

$$
v_{1}(t-\tau)-c_{G} C(t-\tau)\left\langle\langle s\rangle^{-q+1}\right\rangle_{\tau, t} \leqslant \int_{\tau}^{t} \check{v}_{1}(s ; t, \mathbf{x}, \mathbf{v}) d s \leqslant v_{1}(t-\tau)+c_{G} C(t-\tau)\left\langle\langle s\rangle^{-q+1}\right\rangle_{\tau, t} .
$$

Since $\int_{\tau}^{t} \check{v}_{1}(s ; t, \mathbf{x}, \mathbf{v}) d s=(t-\tau)\langle W\rangle_{\tau, t}$ for $\chi_{1}$, we have

$$
v_{1}-c_{G} C\left\langle\langle s\rangle^{-q+1}\right\rangle_{\tau, t} \leqslant\langle W\rangle_{\tau, t} \leqslant v_{1}+c_{G} C\left\langle\langle s\rangle^{-q+1}\right\rangle_{\tau, t} .
$$

That is

$$
\inf _{0 \leqslant \tau \leqslant t}\left(\langle W\rangle_{\tau, t}-c_{G} C\left\langle\langle s\rangle^{-q+1}\right\rangle_{\tau, t}\right) \leqslant v_{1} \leqslant \sup _{0 \leqslant \tau \leqslant t}\left(\langle W\rangle_{\tau, t}+c_{G} C\left\langle\langle s\rangle^{-q+1}\right\rangle_{\tau, t}\right) .
$$

Using the fact that

$$
\sup _{0 \leqslant \tau \leqslant t}\left\langle\langle s\rangle^{-q+1}\right\rangle_{\tau, t} \leqslant \frac{C}{\langle t\rangle}
$$

with $q>2$, we have

$$
\inf _{0 \leqslant \tau \leqslant t}\langle W\rangle_{\tau, t}-\frac{c_{G} C}{\langle t\rangle} \leqslant v_{1} \leqslant \sup _{0 \leqslant \tau \leqslant t}\langle W\rangle_{\tau, t}+\frac{c_{G} C}{\langle t\rangle} .
$$

We next observe that both the collisions and the flow preserve the product structure of the particle density.

Lemma 4.2. The density $f(t, \mathbf{x}, \mathbf{v})$ has the product form $f(t, \mathbf{x}, \mathbf{v})=a\left(t, x_{1}, v_{1}\right) b\left(t, x_{\perp}, v_{\perp}\right)$. In fact, $b\left(t, x_{\perp}, v_{\perp}\right)=b_{0}\left(\breve{v}_{\perp}\left(0 ; t, x_{\perp}, v_{\perp}\right)\right)$.

Proof. Recall that $f_{0}(\mathbf{v})=a_{0}\left(v_{1}\right) b_{0}\left(v_{\perp}\right)$. Next consider any particle $(\mathbf{x}, \mathbf{v})$ at a time $s$ for which $f(s, \mathbf{x}, \mathbf{v})=a\left(s, x_{1}, v_{1}\right) b\left(s, x_{\perp}, v_{\perp}\right)$. Let $t>s$ be times such that there is no collision in the interval $[s, t]$. Then by (2.14) we have

$$
\begin{aligned}
f(s, \mathbf{x}, \mathbf{v}) & =f\left(s, \check{\mathbf{x}}\left(s ; t, x_{1}, v_{1}\right), \check{\mathbf{v}}\left(s ; t, x_{1}, v_{1}\right)\right) \\
& =a\left(s, \check{x}_{1}\left(s ; t, x_{1}, v_{1}\right), \check{v}_{1}\left(s ; t, x_{1}, v_{1}\right)\right) b\left(s, \check{x}_{\perp}\left(s ; t, x_{\perp}, v_{\perp}\right), \check{v}_{\perp}\left(s ; t, x_{\perp}, v_{\perp}\right)\right) .
\end{aligned}
$$

On the other hand, consider a particle that collides at time $\mathrm{t}$ and for which the incoming density has the form $f_{-}(t, \mathbf{x}, \mathbf{v})=a\left(t, x_{1}, v_{1}\right) b\left(t, x_{\perp}, v_{\perp}\right)$. Then

$$
\begin{aligned}
f_{+}(t, \mathbf{x}, \mathbf{v}) & =\int_{\left(u_{1}-W(t)\right)\left(v_{1}-W(t)\right) \leq 0} K\left(v_{1}-W(t), u_{1}-W(t)\right) f_{-}\left(t, \mathbf{x}, u_{1}, v_{\perp}\right) d u_{1} \\
& =a_{+}\left(t, x_{1}, v_{1}\right) b\left(t, x_{\perp}, v_{\perp}\right)
\end{aligned}
$$


where

$$
a_{+}\left(t, x_{1}, v_{1}\right)=\int_{\left(u_{1}-W(t)\right)\left(v_{1}-W(t)\right) \leq 0} K\left(v_{1}-W(t), u_{1}-W(t)\right) a_{-}\left(t, x_{1}, u_{1}\right) d u_{1} .
$$

Thus the product structure is preserved under both the flow and the collisions. The last statement of the lemma is clear because the vertical component is unaffected by the collisions.

Lemma 4.3. As long as $\gamma$ and $c_{G}$ are small enough, we have

$$
\left|R_{W}(t)\right| \leq C \frac{\left(A \gamma^{p+1}+\gamma+c_{G}\right)^{p+1}}{(1+t)^{p+1}}
$$

where $C$ is independent of $t, \gamma, c_{G}$ and $A$.

With a slightly finer proof, one can prove the better rate of decay $\left|R_{W}(t)\right| \leq C \frac{\left(A \gamma^{p+1}+\gamma+c_{G}\right)^{p+1}}{(1+t)^{p+d}}$. However, by (2.9) we have $\sigma<p+1$ and hence $\sigma$ is a slower decay rate. So we will only bother to prove the $p+1$ decay rate for $\left|R_{W}(t)\right|$.

Proof. Without loss of generality, we may consider only the right side of the body because the left side provides the same decay rate with the same proof. We begin by proving an upper bound on $f_{+}$. We claim that

$$
b_{0}\left(\check{v}_{\perp}\left(0 ; t, x_{\perp}, v_{\perp}\right)\right) a_{+}^{*} \leqslant 2 C_{2} b_{0}\left(\check{v}_{\perp}\left(0 ; t, x_{\perp}, v_{\perp}\right)\right)
$$

where

$$
a_{+}^{*}=\sup \left\{a_{+}\left(\tau, \xi ; u_{1}\right) \mid \xi=X(\tau), \tau \in[0, \infty) \text {, and } u_{1} \in\left[V_{\infty}-3 \gamma, V_{\infty}+3 \gamma\right]\right\} .
$$

Assuming $(\mathbf{x}, \mathbf{v}) \notin Z(t)$, let $t_{0}=t_{0}(t, \mathbf{x}, \mathbf{v})$ be the first collision time after time $t$ and $\tau(t, \mathbf{x}, \mathbf{v})$ be the last collision time before time $t$. We use (4.1) to split

$$
\begin{aligned}
f_{+}(t, \mathbf{x}, \mathbf{v})= & \int_{u_{1} \leqslant W(t)} K\left(v_{1}-W(t), u_{1}-W(t)\right) \chi_{1}\left(t, u_{1}, v_{\perp}\right) \\
& \times f_{+}\left(\tau, \check{\mathbf{x}}\left(\tau ; t, \mathbf{x}, u_{1}, v_{\perp}\right), \check{v}_{1}\left(\tau ; t, \mathbf{x}, u_{1}, v_{\perp}\right), \check{v}_{\perp}\left(\tau ; t, \mathbf{x}, u_{1}, v_{\perp}\right)\right) d u_{1} \\
& +\int_{u_{1} \leqslant W(t)} K\left(v_{1}-W(t), u_{1}-W(t)\right) \chi_{0}\left(t, u_{1}, v_{\perp}\right) \\
& \times f_{0}\left(\check{v}_{1}\left(0 ; t, \mathbf{x}, u_{1}, v_{\perp}\right), \check{v}_{\perp}\left(0 ; t, \mathbf{x}, u_{1}, v_{\perp}\right)\right) d u_{1} \\
= & I+I I
\end{aligned}
$$

and have

and

$$
\left|v_{1}-\check{v}_{1}\left(t_{0} ; t, x_{1}, v_{1}\right)\right| \leqslant c_{G}
$$

$$
\inf _{s \leqslant t_{0}}\langle W\rangle_{s, t_{0}}-\frac{c_{G} C}{\left\langle t_{0}\right\rangle} \leqslant \check{v}_{1}\left(t_{0} ; t, x_{1}, v_{1}\right) \leqslant \sup _{s \leqslant t_{0}}\langle W\rangle_{s, t_{0}}+\frac{c_{G} C}{\left\langle t_{0}\right\rangle} .
$$

Here, (4.6), which we have used many times, comes from (2.13) and (4.7) comes from Lemma 4.1. We deduce from (4.6) and (4.7) that

$$
\left|v_{1}-W(t)\right| \leqslant 2\left(\gamma+c_{G}\right)<3 \gamma
$$

because we assumed $\gamma>2 c_{G}$.

We can now estimate (4.5). By Lemma 4.2

$$
\begin{aligned}
I= & \int_{u_{1} \leqslant W(t)} K\left(v_{1}-W(t), u_{1}-W(t)\right) \chi_{1}\left(t, u_{1}, v_{\perp}\right) \\
& \times a_{+}\left(\tau, \check{x}\left(\tau ; t, x_{1}, u_{1}\right), \check{v}_{1}\left(\tau ; t, x_{1}, u_{1}\right)\right) b_{0}\left(\check{v}_{\perp}\left(0 ; t, x_{\perp}, v_{\perp}\right)\right) d u_{1}
\end{aligned}
$$

and

$$
\begin{aligned}
I I= & \int_{u_{1} \leqslant W(t)} K\left(v_{1}-W(t), u_{1}-W(t)\right) \chi_{0}\left(t, u_{1}, v_{\perp}\right) \\
& \times a_{0}\left(\check{v}_{1}\left(0 ; t, x_{1}, u_{1}\right)\right) b_{0}\left(\check{v}_{\perp}\left(0 ; t, x_{\perp}, v_{\perp}\right)\right) d u_{1} .
\end{aligned}
$$

For $I$, we use Lemma 4.1 to deduce

$$
I \leqslant b_{0}\left(\check{v}_{\perp}\left(0 ; t, x_{\perp}, v_{\perp}\right)\right) a_{+}^{*} \int_{\inf _{\tau \leqslant t}\langle W\rangle_{\tau, t}-\frac{c_{G} C}{\langle t\rangle}}^{W(t)} K\left(v_{1}-W(t), u_{1}-W(t)\right) d u_{1} .
$$


From the definition of $\mathcal{W}$, we deduce

$$
\begin{aligned}
W(t) & \leqslant V_{\infty}+\frac{\left(C \gamma+A \gamma^{p+1}\right)}{(1+t)^{\sigma}} \leqslant V_{\infty}+C A \gamma \\
\inf _{0<s<t}\langle W\rangle_{s, t} & \geq V_{\infty}-\frac{1}{t} \int_{0}^{t} \frac{\left(C \gamma+A \gamma^{p+1}\right)}{(1+s)^{\sigma}} d s \geqslant V_{\infty}-C A \gamma .
\end{aligned}
$$

because $A \geqslant 1$. Hence

$$
I \leqslant b_{0}\left(\check{v}_{\perp}\left(0 ; t, x_{\perp}, v_{\perp}\right)\right) C_{1} A\left(\gamma+c_{G}\right) a_{+}^{*}
$$

because $K$ is bounded.

For the second term of (4.5), we know as before that $\left|\check{v}_{1}\left(0 ; t, X(t), u_{1}\right)-u_{1}\right|<C$. Hence

$$
I I \leqslant b_{0}\left(\check{v}_{\perp}\left(0 ; t, x_{\perp}, v_{\perp}\right)\right) C \int_{u_{1} \leqslant W(t)} K\left(v_{1}-W(t), u_{1}-W(t)\right)\left\langle u_{1}\right\rangle^{-l_{1}} d u_{1}
$$

since $a_{0}\left(v_{1}\right) \leqslant C\left\langle v_{1}\right\rangle^{-l_{1}}$. Noticing (4.8), we use condition (2.7) to deduce that

$$
I I \leqslant C b_{0}\left(\check{v}_{\perp}\left(0 ; t, x_{\perp}, v_{\perp}\right)\right) \text { for } v_{1} \in\left[V_{\infty}-2 \gamma, V_{\infty}+2 \gamma\right]
$$

Thus (4.5) becomes

$$
f_{+}(t, \mathbf{x}, \mathbf{v}) \leqslant b_{0}\left(\check{v}_{\perp}\left(0 ; t, x_{\perp}, v_{\perp}\right)\right)\left\{C_{1} A\left(\gamma+c_{G}\right) a_{+}^{*}+C_{2}\right\}
$$

Using Lemma 4.2 on the left side of this inequality as well, we have

$$
\begin{aligned}
& a_{+}\left(\tau, \check{x}_{1}\left(\tau ; t, x_{1}, u_{1}\right), \check{u}_{1}\left(\tau ; t, x_{1}, u_{1}\right)\right) b_{0}\left(\check{v}_{\perp}\left(0 ; t, x_{\perp}, v_{\perp}\right)\right) \\
\leqslant & b_{0}\left(\check{v}_{\perp}\left(0 ; t, x_{\perp}, v_{\perp}\right)\right)\left\{C_{1} A\left(\gamma+c_{G}\right) a_{+}^{*}+C_{2}\right\}
\end{aligned}
$$

so that

$$
\begin{aligned}
b_{0}\left(\check{v}_{\perp}\left(0 ; t, x_{\perp}, v_{\perp}\right)\right) a_{+}^{*} \leqslant \quad & b_{0}\left(\check{v}_{\perp}\left(0 ; t, x_{\perp}, v_{\perp}\right)\right) C_{1} A\left(\gamma+c_{G}\right) a_{+}^{*} \\
& +C_{2} b_{0}\left(\check{v}_{\perp}\left(0 ; t, x_{\perp}, v_{\perp}\right)\right) .
\end{aligned}
$$

So choosing $\gamma$ so small that $A\left(\gamma+c_{G}\right)<\left(2 C_{1}\right)^{-1}$, we have proven the claim (4.3).

We now estimate $R_{W}^{R}(t)$. By (4.1), we have

$$
\begin{aligned}
& \left|f_{-}(t, \mathbf{x}, \mathbf{v})-f_{N B}(t, \mathbf{x}, \mathbf{v})\right| \\
= & \left|\chi_{1}(t, \mathbf{v}) f_{+}(\tau, \check{\mathbf{x}}(\tau ; t, \mathbf{x}, \mathbf{v}), \check{v}(\tau ; t, \mathbf{x}, \mathbf{v}))+\chi_{0}(t, \mathbf{v}) f_{0}(\check{v}(0 ; t, \mathbf{x}, \mathbf{v}))-f_{N B}(t, \mathbf{x}, \mathbf{v})\right| \\
= & \chi_{1}(t, \mathbf{v})\left|f_{+}(\tau, \check{\mathbf{x}}(\tau ; t, \mathbf{x}, \mathbf{v}), \check{v}(\tau ; t, \mathbf{x}, \mathbf{v}))-f_{N B}(t, \mathbf{x}, \mathbf{v})\right| \\
= & \chi_{1}(t, \mathbf{v}) b_{0}\left(\check{v}_{\perp}\left(0 ; t, x_{\perp}, v_{\perp}\right)\right)\left|a_{+}\left(\tau, \check{x}_{1}\left(\tau ; t, x_{1}, v_{1}\right), \check{v}_{1}\left(\tau ; t, x_{1}, v_{1}\right)\right)-a_{0}\left(\check{v}_{1}\left(0 ; t, x_{1}, v_{1}\right)\right)\right| \\
\leqslant & C \chi_{1}(t, \mathbf{v}) b_{0}\left(\check{v}_{\perp}\left(0 ; t, x_{\perp}, v_{\perp}\right)\right)
\end{aligned}
$$

where in the last line, we used (4.3). Hence

$$
\begin{aligned}
\left|R_{W}^{R}(t)\right| & \leqslant \int_{D(t)} \int_{v_{1} \leqslant W(t)} L\left(v_{1}-W(t)\right)\left|f_{-}(t, \mathbf{x}, \mathbf{v})-f_{N B}(t, \mathbf{x}, \mathbf{v})\right| d \mathbf{v} \\
& \leqslant C \int_{D(t)} \int_{v_{x} \leqslant W(t)} L\left(v_{1}-W(t)\right) \chi_{1}(t, \mathbf{v}) b_{0}\left(\check{v}_{\perp}\left(0 ; t, x_{\perp}, v_{\perp}\right)\right) d \mathbf{v} \\
& \leqslant C \int_{D(t)} \int b_{0}\left(v_{\perp}\right) d v_{\perp} \int_{\inf _{\tau \leqslant t}\langle W\rangle_{\tau, t}-\frac{c_{G} C}{\langle t\rangle}}^{W(t)} L\left(v_{1}-W(t)\right) d v_{1} \\
& \leqslant C \int_{\inf _{\tau \leqslant t}\langle W\rangle_{\tau, t}-\frac{c_{G} C}{\langle t\rangle}}^{W(t)} L\left(v_{1}-W(t)\right) d v_{1}
\end{aligned}
$$


Recalling estimate (4.9), we then have by assumption on $K$ that

$$
\begin{aligned}
\left|R_{W}^{R}(t)\right| & \left.\leqslant C \int_{V_{\infty}-\frac{1}{t} \int_{0}^{t} \eta(s) d s-\frac{c_{G} C}{\langle t\rangle}}^{W(t)} \mid v_{1}-W(t)\right)\left.\right|^{p} d v_{1} \\
& \leqslant C\left|V_{\infty}-\frac{1}{t} \int_{0}^{t} \frac{\left(C \gamma+A \gamma^{p+1}\right)}{(1+s)^{\sigma}} d s-W(t)-\frac{c_{G} C}{\langle t\rangle}\right|^{p+1} \\
& \leqslant C \frac{\left(A \gamma^{p+1}+\gamma+c_{G}\right)^{p+1}}{(1+t)^{p+1}}
\end{aligned}
$$

where in the last line we used the fact that

$$
\frac{1}{t} \int_{0}^{t} \frac{\gamma+A \gamma^{p+1}}{\langle s\rangle^{\sigma}} d s \leqslant C \frac{\gamma+A \gamma^{p+1}}{\langle t\rangle}
$$

\section{Proof of Theorem 2.1}

We begin with the following elementary lemma.

Lemma 5.1. Suppose

$$
\frac{d Y}{d t}=-b(t) Y+d(t)
$$

for $t \geq 0$, where $b(t) \geq b_{0}>0$ and $|d(t)| \leq C_{0}(1+t)^{-\sigma}$ with $\sigma>1$. Then there exists $C_{1}$ such that

$$
|Y(t)| \leqslant|Y(0)| e^{-b_{0} t}+C_{1}(1+t)^{-\sigma}
$$

where $C_{1}=O\left(C_{0}\right)$ as $C_{0} \rightarrow 0$.

Proof. Let $B(t)=\int_{0}^{t} b(s) d s$. Then $\frac{d}{d t}\left[e^{B(t)} Y(t)\right]=e^{B(t)} d(t)$ so that

$$
Y(t)=e^{-B(t)} Y(0)+e^{-B(t)} \int_{0}^{t} e^{B(s)} d(s) d s
$$

Thus

$$
|Y(t)| \leq|Y(0)| e^{-b_{0} t}+\int_{0}^{t} e^{-b_{0}(t-s)} C_{0}(1+s)^{-\sigma} d s .
$$

Estimating $(1+s)^{-\sigma} \leq 1$ in $[0, t / 2]$ and $(1+s)^{-\sigma} \leq(1+t / 2)^{-\sigma}$ in $[t / 2, t]$, we find that

$$
|Y(t)| \leq|Y(0)| e^{-b_{0} t}+\frac{C_{0}}{b_{0}} e^{-b_{0} t / 2}+\frac{C_{0}}{b_{0}}\left(1+\frac{t}{2}\right)^{-\sigma} .
$$

Lemma 5.2. There exists a constant $A$ such that for small enough $\gamma$ and $c_{G}$, we have

In other words, $V_{W} \in \mathcal{W}$.

$$
\left|V_{W}(t)-V_{\infty}\right|<\gamma e^{-b_{0} t}+\frac{A \gamma^{p+1}}{\langle t\rangle^{\sigma}} .
$$

Proof. We define

and

$$
Y(t)=V_{W}(t)-V_{\infty}, \quad b(t)=\frac{F_{00}(W(t))-F_{00}\left(V_{\infty}\right)}{W(t)-V_{\infty}}
$$

$$
d(t)=R_{W}(t)-F_{00}(W(t))+F_{0}(t, X(t), W(t)) .
$$

We have $|Y(0)|=\gamma$ by definition. From Theorem 3.1 and Lemma 4.3 , we have

$$
|d(t)| \leqslant c_{G} C\langle t\rangle^{-\sigma}+C\left(A \gamma^{p+1}+\gamma+c_{G}\right)^{p+1}\langle t\rangle^{-1-p} .
$$

Since $\sigma \leqslant 1+p$ by its definition (2.9), we have

$$
|d(t)| \leqslant C\left(c_{G}+\left(A \gamma^{p+1}+\gamma+c_{G}\right)^{p+1}\right)\langle t\rangle^{-\sigma} .
$$

Now we apply Lemma 5.1 to obtain

$$
\left|V_{W}(t)-V_{\infty}\right| \leqslant \gamma e^{-b_{0} t}+C\left(c_{G}+\left(A \gamma^{p+1}+\gamma+c_{G}\right)^{p+1}\right)\langle t\rangle^{-\sigma} .
$$


We choose $A>2 C$ and $c_{G}<\frac{1}{2} \gamma^{2 p+1}$. We then have

$$
C\left(c_{G}+\left(A \gamma^{p+1}+\gamma+c_{G}\right)^{p+1}\right)<C \gamma^{p+1}\left(\frac{1}{2} \gamma^{p}+\left(A \gamma^{p}+1+\frac{1}{2} \gamma^{2 p}\right)^{p+1}\right)<A \gamma^{p+1}
$$

by choosing $\gamma$ small enough, since $p>0$. That is,

$$
\left|V_{W}(t)-V_{\infty}\right|<\gamma e^{-b_{0} t}+\frac{A \gamma^{p+1}}{\langle t\rangle^{\sigma}}
$$

as claimed.

We now have

$$
\frac{d V_{W}}{d t}=\frac{E-F_{0}(W)}{V_{\infty}-W}\left(V_{\infty}-V_{W}\right)+d(t)
$$

with $V_{W}(0)=V_{0}$ and $\gamma=\left|V_{0}-V_{\infty}\right|$. Recall that $W \in \mathcal{W}$ means that $W(\cdot)$ is Lipschitz, $W(0)=V_{0}$ and $\left|W(t)-V_{\infty}\right| \leq \gamma e^{-b_{0} t}+\frac{A \gamma^{p+1}}{\langle t\rangle^{\sigma}}$. Given $L>0$, define

$$
\mathcal{K}=\{W \in \mathcal{W} \mid \operatorname{esssup}(|W(t)|+|\dot{W}(t)|) \leq L\}
$$

which is a convex and compact subset of $C_{b}([0, \infty))$. Define the operator $\mathcal{A}$ on $\mathcal{K}$ by $\mathcal{A}(W)=V_{W}$. A choice of $L$ sufficiently large implies that $\mathcal{A}: \mathcal{K} \rightarrow \mathcal{K}$. In fact,

$$
\begin{aligned}
\left|V_{W}(t)\right| & \leqslant V_{\infty}+3 \gamma \\
\left|\dot{V}_{W}(t)\right| & \leqslant 3 \gamma_{V \in\left[V_{\infty}-3 \gamma, V_{\infty}+3 \gamma\right]} \max _{00}^{\prime}(V)+C\left(\left(\gamma+A \gamma^{p+1}+c_{G}\right)^{p+1}+c_{G}\right)
\end{aligned}
$$

as already shown in the proof of Lemma 5.2. The next lemma will allow us to apply the Schauder fixed point theorem to deduce that $\mathcal{A}$ has a fixed point, which will complete the proof of existence in Theorem 2.1

Lemma 5.3. If we provide $\mathcal{K}$ with the topology of $C_{b}([0, \infty))$, then $\mathcal{A}$ is a continuous operator.

Proof. We follow the proof in [9, Lemma 7.1], slightly modified. The term $H=H_{W}=F_{00}(W)-$ $F_{0}(t, X, W)$ involves no collisions at all, while the recollision force satisfies $\left|R_{W}\right| \leqslant C \gamma\langle t\rangle^{-\sigma} \leqslant C \gamma$.

For any $t>0$, we define $B_{W}^{N}(t)$ as the set of $(\mathbf{x}, \mathbf{v})$ such that the trajectory passing through $(t, \mathbf{x}, \mathbf{v})$ has collided with the body at least $N+1$ times in $[0, t]$, and we define $A_{W}^{N}(t)$ as its complement. Using the estimate (4.2) that $\sup _{t \in[0, \infty), W \in \mathcal{W}}\left|R_{W}(t)\right| \leq C \gamma$ and iterating this estimate $N$ times, we have

$$
\sup _{t \in[0, \infty), W \in \mathcal{W}}\left|R_{W}\left(t ; B_{W}^{N}(t)\right)\right| \leq(C \gamma)^{N} .
$$

For any $\epsilon>0$, this expression is at most $\frac{\epsilon}{4}$ by choosing $\gamma<1 / C$ and $N=N_{\epsilon}$ large enough.

Now let $W_{j} \rightarrow W$ in $\mathcal{K}$. Given any time $S>0$, we may write

$$
\begin{aligned}
& \sup _{0 \leq t<\infty}\left|R_{W_{j}}(t)-R_{W}(t)\right| \\
\leq & \sup _{S \leq t<\infty}\left|R_{W_{j}}(t)-R_{W}(t)\right|+\sup _{0 \leq t<S}\left|R_{W}\left(t ; B_{W}^{N}(t)\right)\right|+\sup _{0 \leq t<S}\left|R_{W_{j}}\left(t ; B_{W_{j}}^{N}(t)\right)\right| \\
& +\sup _{0 \leq t<S}\left|R_{W_{j}}\left(t ; A_{W_{j}}^{N}(t)\right)-R_{W}\left(t ; A_{W}^{N}(t)\right)\right| \\
= & I+I I+I I I+I V .
\end{aligned}
$$

By estimate (4.2), we may choose $S=S_{\varepsilon}$ so large that $|I|<\epsilon / 4$. By estimate (5.1), we have $|I I|+|I I I| \leq$ $2 \epsilon / 4$.

Now in $I V$ there are no more than $N$ collisions. Therefore we can express both terms in $I V$ as iterates of at most $N$ integrals by repeated use of the collision boundary condition. The resulting finite number of iterated integrals contain $W_{j}$ in a finite number of places in the expression $R_{W_{j}}\left(t ; A_{W_{j}}^{N}(t)\right)$. (See Lemma 2.2.) Therefore they converge as $j \rightarrow \infty$ to the same expression with $W_{j}$ replaced by $W$, and the convergence is uniform for $t \in[0, S]$. Thus we can choose $j$ so large that $|I V|<\epsilon / 4$. So we conclude that $R_{W_{j}}(t) \rightarrow R_{W}(t)$ in $C_{b}([0, \infty))$.

It is also clear that

$$
H_{W_{j}} \rightarrow H_{W}
$$


in $\mathcal{K}$ because no collisions are involved. It follows from the equation (2.18) for $V_{W}(t)$ that $\mathcal{A}$ is continuous in the topology of $C_{b}([0, \infty))$.

So far we have proven the existence part of Theorem 2.1. We now prove that every solution in this sense satisfies (2.10) with a simple argument. In fact, consider any solution $(\tilde{V}(t), \tilde{f})$ that satisfies

$$
\left|\tilde{V}(0)-V_{\infty}\right|=\gamma<\gamma+A \gamma^{p+1}
$$

Then

$$
\left|\tilde{V}(t)-V_{\infty}\right|<\gamma e^{-b_{0} t}+\frac{A \gamma^{p+1}}{\langle t\rangle^{\sigma}}
$$

for small $t$. Now suppose we have equality at some later time. Let $T$ be the earliest such time. Then the existence of such a time $T$ contradicts Lemma 5.2 because $(\tilde{V}(t), \tilde{f})$ is a fixed point of the mapping $\mathcal{A}$. Thus (5.2) is valid for all $t<\infty$.

\section{REFERENCES}

[1] K. Aoki, G. Cavallaro, C. Marchioro, and M. Pulvirenti, On the motion of a body in thermal equilibrium immersed in a perfect gas, ESAIM: Mathematical Modelling and Numerical Analysis 42 (2008), 263-275.

[2] K. Aoki and F. Golse, On the speed of approach to equilibrium for a collisionless gas, Kinet. Relat. Models 4 (2011), $87-107$.

[3] K. Aoki, T. Tsuji, and G. Cavallaro, Approach to steady motion of a plate moving in a free-molecular gas under a constant external force, Phys. Rev. E 80 (2009), 016309.

[4] P. Buttà, G. Cavallaro, and C Marchioro, Motion of a body immersed in a Vlasov system, Lecture Notes in Mathematics, 2135 (2015), 63-100.

[5] S. Caprino, G. Cavallaro, and C. Marchioro, On a microscopic model of viscous friction, Math. Models Meth. Appl. Sci. 17 (2007), 1369-1403.

[6] S. Caprino, C. Marchioro, and M. Pulvirenti, Approach to equilibrium in a microscopic model of friction, Commun. Math. Phys. 264 (2006), 167-189.

[7] G. Cavallaro, On the motion of a convex body interacting with a perfect gas in the mean-field approximation, Rend. Mat. Appl. 27 (2007), 123-145.

[8] G. Cavallaro and C. Marchioro, On the Motion of an Elastic Body in a Free Gas, Reports on Mathematical Physics 69 (2012), 251-264.

[9] X. Chen and W. Strauss, Approach to Equilibrium of a Body Colliding Specularly and Diffusely with a Sea of Particles, Arch. Rational Mech. Anal. 211 (2014), 879-910.

[10] X. Chen and W. Strauss, Velocity Reversal Criterion of a Body Immersed in a Sea of Particles, Commun. Math. Phys. 338 (2015), 139-168.

[11] R. Glassey, The Cauchy Problem in Kinetic Theory, SIAM, Philadelphis, PA, 1996.

[12] Y. Guo, Decay and continuity of the Boltzmann equation in bounded domains, Arch. Rational Mech. Anal. 197 (2010), 713-809.

[13] F.Sisti and C. Ricciuti, Effects of concavity on the motion of a body immersed in a Vlasov gas, SIAM J. Math. Anal. 46 (2014), 3759-3611

[14] Y. Sone, Kinetic Theory and Fluid Dynamics, Birkhäuser, 2002.

[15] H. Spohn, Large Scale Dynamics of Interacting Particles, Springer, 1991.

[16] T. Tsuji and K. Aoki, Decay of an oscillating plate in a free-molecular gas, in: Rarefied Gas Dynamics, D. A. Levin et al. eds., AIP, Melville, 2011, 140-145.

[17] T. Tsuji and K. Aoki, Moving boundary problems for a rarefied gas: spatially one-dimensional case, J. Comput. Phys. 250 (2013), 574-600.

[18] T. Tsuji, J. Arai and S. Kawano, Slow approach to steady motion of a concave body in a free-molecular gas, Phys. Rev. E 92 (2015), 012130.

Department of Mathematics, University of Rochester, Rochester, NY 14627

E-mail address: chenxuwen@math.brown.edu

URL: http://www.math.rochester.edu/people/faculty/xchen84/

Department of Mathematics and Lefschetz Center for Dynamical Systems, Brown University, Providence, RI 02912

E-mail address: wstrauss@math.brown.edu

URL: http://www. math.brown. edu/ wstrauss/ 